\title{
PRIMEROS AUXILIOS EN DECOMISOS DE ANIMALES DE VENTA O TRÁFICO ILEGAL
}

\section{Enrique Rimbaud}

Doctor en medicina y tecnología veterinaria,FCA-UCC, erimbaud@gmail.com

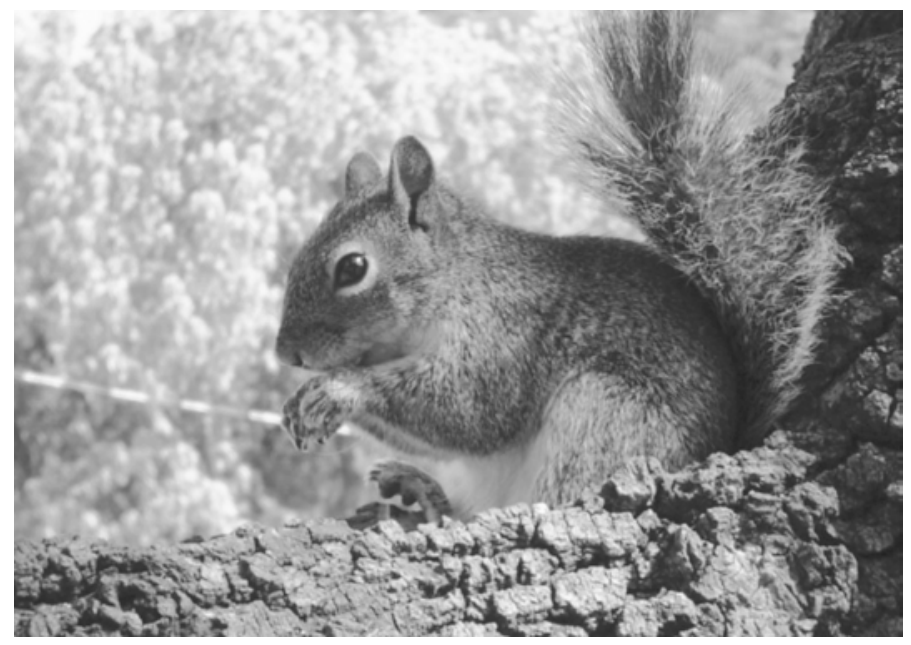

$\mathrm{M}$ uy a pesar nuestro los decomisos de animales en venta o tráfico ilegal son cada vez más frecuentes y deberían de ser cada vez más frecuentes, hasta un día acabar con la depredación irresponsable en la que lamentablemente está implicada mucha gente e intereses económicos.

Generalmente los individuos que trafican o venden animales ilegalmente no se preocupan en absoluto de su bienestar, salud, correcta alimentación ni higiene. Incluso llegan a modificar diferentes características del animal para que sean más atractivos para la venta (chocoyos que se pintan de amarillo en la cabeza para semejar loras nuca amarilla), o hacer más seguro, según ellos, su manejo (iguanas, garrobos, serpientes, etc. que les cosen la boca para que no muerdan). Otras veces mutilan o deforman parte del animal para facilitar su manejo o almacenamiento (loras o lapas con las alas cortadas, lapas con sus plumas de la cola enrolladas con sellador).

A nivel de mamíferos debemos separar aquellas especies de valor comercial como mascota o atracción zoológica (monos, ardillas, tigrillos), en los que generalmente matan a la madre para comercializar sus crías, de aquellos que se comercializan por sus propiedades medicinales o atributos gastronómicos (cusucos, garrobos, iguanas, etc.) que generalmente se venden ejemplares adultos sin importar el sexo o el estado fisiológico del animal, enfrentando muchas veces el decomiso de hembras preñadas o cargadas de huevos.
Todo esto nos lleva indudablemente a que cuando enfrentamos el tratamiento médico de animales decomisados debemos encarar dos grandes capítulos además de la emergencia inherente, estos son el manejo, estos son el manejo del estrés agudo, y la necesidad de una rehabilitación posterior previa a la devolución de los animales a su medio natural.

Organización de la atención veterinaria. Hay dos cosas fundamentales previas a llevar a cabo el examen médico o intervenciones quirúrgicas pertinentes, estas son:

Asegurarnos de contar en el más breve plazo posible con comida suficiente en cantidad y calidad para todas las especies decomisadas y de acuerdo a sus requerimientos específicos. Si bien en el estrés reinante muchos de los animales a tratar pueden negarse en primera instancia a comer, lo mas relajante que hay para los animales es notar que cuentan con comida abundante.

Debemos contar con instalaciones (jaulas, terrarios, peceras, etc.) en cantidad suficiente para brindarles el espacio necesario a los animales sin causarles estrés, y que estas no dañen a los animales.

Es importante contar con agua en cantidades suficientes y observar las más estrictas medidas de higiene y biodiversidad en todo momento.

Como no sabemos la condición o conducta (agravada y transformada por el estrés), de estos animales, 
es necesario contar con suficiente cantidad de cuerdas, lazos mecánicos, guantes de seguridad y eventualmente una buena diversidad de sedantes y tranquilizantes adecuados para cada especie.

La atención veterinaria. Luego de ubicar los animales en sus habitáculos respectivos, limpios, espaciosos y con agua y comida suficiente, procederemos a la atención veterinaria.

La misma la dividiremos en las siguientes etapas:

Manejo del estrés. El manejo adecuado de estrés es sin lugar a dudas la clave del éxito en el tratamiento y recuperación de los animales decomisados. Muchos de los animales han pasado por un verdadero periodo de terror y maltrato, incluso siendo muchos de ellos heridos y abusados.

Sabemos que el estrés es causado por la deplección catecolaminica de las glándulas adrenales, y que esto lleva desde un simple estado de alerta, a la angustia y tensión máximas, pasando fácilmente al estado de shock, pudiendo muchos de estos animales morir en ese instante por paro cardiorespiratorio o choque neurogénico grave.

La administración de glucocorticoides (dexametasona, betametasona, flumetasona, etc.) disminuyen francamente la condición de estrés del animal previniendo la aparición del estado de shock.

Manejo de Urgencia y Emergencia. Luego del manejo exitoso del estrés, debemos encarar el manejo inmediato de la condición patológica primaria del animal. Debemos revisar la presencia de heridas y otros traumas (fracturas, contusiones, desgarres, abrasiones, hematomas, etc.).

A esto debemos darle atención primaria inmediatamente dado que muchas de estas patologías quirúrgicas pueden comprometer gravemente la condición física del animal.

Debemos controlar estrictamente la hidratación del animal evaluando la necesidad o no de establecer fluidoterapia endovenosa o subcutánea de acuerdo a las necesidades que detectemos en el animal.

Este es el momento en el cual debemos decidir la necesidad de intervenciones quirúrgicas dado que hacerlo posterior a esta instancia solo serviría para agravar la condición de estrés.

La revisación de los animales y la aplicación de medicamentos debe hacerse necesariamente fuera de la jaula para que el animal no asocie la jaula con el dolor.

La utilización de vendajes debe ser una decisión estudiada dado que los vendajes muchas veces molestan o estresan al animal más de lo que ayudan, de preferencia, solo usarlos en heridas quirúrgicas con suturas muy grandes o complejas, o en abrasiones y quemaduras muy extensas.

Estudio de la condición clínica. Luego del manejo del estrés, la urgencia y emergencia realizamos el examen clínico normal para determinar la condición clínica exacta del animal, presencia de ecto o endoparásitos, enfermedades infecciosas y/o zoonóticas, estado nutricional general, instalando la terapéutica necesaria, y redactando en esta instancia el informe general y particular que podrá servir a las autoridades intervinientes en el decomiso como datos de peritaje e informe médico exigidos para el expediente del caso.

Tabla 1. Datos fisiológicos normales de mamíferos comúnmente decomisados

\begin{tabular}{lllcr}
\hline $\begin{array}{l}\text { Nombre } \\
\text { Común }\end{array}$ & $\begin{array}{l}\text { Nombre } \\
\text { Científico }\end{array}$ & $\begin{array}{l}\text { Frecuencia } \\
\text { respiratoria } \\
\text { (Respiraciones } \\
\text { Por minuto) }\end{array}$ & $\begin{array}{l}\text { Frecuencia } \\
\text { Circulatoria } \\
\text { (Pulsaciones } \\
\text { por minuto) }\end{array}$ & $\begin{array}{c}\text { Temperatura } \\
\text { Rectal }\left({ }^{\circ} \mathrm{C}\right)\end{array}$ \\
\hline Tigrillo & Leopardus Tigrinus & $18-36$ & $100-150$ & $38-38.5$ \\
Jaguar & Felis onca & $8-24$ & $70-140$ & $37-39.5$ \\
Armadillo & & & & \\
Común o cusuco & Dasipus novemincintus & $25-56$ & $70-120$ & $32.7-35.3$ \\
Mono araña & Ateles geoffroyi & $20-50$ & $95-112$ & $37.2-40.2$ \\
Mono carablanca & Cebus capucinus & & & \\
Mono congo & Alowatta palliata & & & \\
$\begin{array}{l}\text { Danto } \\
\text { Chiza o ardilla }\end{array}$ & $\begin{array}{l}\text { Tapirus bairdii } \\
\text { Sciurus variegatoides }\end{array}$ & $42-104$ & $230-380$ & $36.4-37.2$ \\
Venado & Odocoilus virginianus & $50-70$ & $80-100$ & $39-41$ \\
Sahino & Pecari tajacu & $40-70$ & $70-100$ & $37.5-39$ \\
\hline
\end{tabular}


Rehabilitación. Luego de haber atendido las necesidades primarias de la salud, alimentación y cuidados generales de los animales debemos encarar la rehabilitación psicosomática y psicobiológica de estos.

\section{Esto pasa por dos o tres aspectos:}

- Mantenimiento de las condiciones de higiene, de las instalaciones, cantidad y calidad de la comida, evi- tando el estrés en las condiciones cuando estas son necesarias.

- En el casi de los mamíferos y aves bebes, establecer dietas adecuadas para cada especie.

- Debemos estar seguros que los animales están en buen estado de salud y que puedan valerse por sí mismos antes de liberarlos al ambiente natural donde ellos viven.

\section{REFERENCIAS BIBLIOGRÁFICAS}

Boily, P. Individual variation in metabolic traits of wild nine-banded armadillos (Dasypus novemcinctus), and the aerobic capacity model for the evolution of endothermy , Department of Biological Sciences, University of New Orleans, New Orleans, LA 70148, USA

Cuba Caparó, A. 1976. Some hematologic and temperature determinations in the 7-banded armadillo (Dasypus hybridus)., Lab. Anim. Sci, Jun;26(3):450-5

Gual Sill, F. 2005. Medicine and Management of Pet Primates, 30 World Congress of World Small Animal Veterinary Association, Primates, México,

Guerra Centeno, D. Valores de referencia para morfometría y fisiología del pecarí de labios blancos (Tayassu pecari): efectos del sexo, categoría de peso y población

http://www.veterinaria.org/revistas/redvet/n080807/080704.pdf

Hernandez-Divers SM; Gammonds, D; Michael Conner, L. Mengak, M. Hernandez-Divers, SJ. 2006. Anesthetic protocol, surgical procedure and disease Investigation of a population of Nine-banded armadillos (Dasypus novemcinctus) European Association of Zoo- and Wildlife Veterinarians (EAZWV) 6th scientific meeting, May 24 - 28 - Budapest, Hungary

Janssen, BA; Rideout, DL; Edwards, MS. 1999. Tapir medicine. In: Fowler, M. E., and R. E. Miller (eds.). Zoo and Wild Animal Medicine: Current Therapy 4. W. B. Saunders Co., Philadelphia, Pennsylvania. Pp. 562-563

Kauffman, G. Rodent and Small Animal Medicine, in Zoological Medicine, http://ocw.tufts.edu/Content/5/ readings/215920

White; Seymour. 2003 Mammalian basal metabolic rate is proportional to body mass ${ }^{2 / 3}$, Proc. Natl. Acad. Sci. USA, 10.1073/pnas.0436428100

http://www.panthera.org/documents/JagHealthProgramManualJune2005_english.pdf 